\title{
Yang-Mills Equations on the Two-Dimensional Sphere
}

\author{
Th. Friedrich and L. Habermann \\ Sektion Mathematik Humboldt Universität, DDR-1086 Berlin, PSF 1297, \\ German Democratic Republic
}

\begin{abstract}
We construct a 1:1 correspondence between the equivalence classes of Yang-Mills fields over $S^{2}$ and the conjugacy classes of closed geodesics of the structure group. Furthermore, we give an explicit isolation theorem for any Yang-Mills field over $S^{2}$.
\end{abstract}

Let $G$ be a compact and connected Lie group and consider a principal fibre bundle $(P, M, G)$ over a compact oriented Riemannian manifold $M$. On the space $\mathscr{C}(P)$ of connections on $P$ the Yang-Mills functional $L: \mathscr{C}(P) \rightarrow R$ is defined by

$$
L(Z)=\int_{M}\left\|\Omega_{Z}\right\|^{2} d M
$$

where $\Omega_{Z}$ is the curvature of the connection $Z$ and the norm is defined by the Riemannian metric on $M$ and a fixed Ad-invariant scalar product on the Lie algebra of $G$. The critical points of the Yang-Mills functional are called Yang-Mills connections. It is well-known (see [3]) that $Z \in \mathscr{C}(P)$ is a Yang-Mills connection if and only if $Z$ is a solution of the Yang-Mills equation $D_{Z} * \Omega_{Z}=0$. In the introduction of [2] Atiyah and Bott mentioned that, for the 2-sphere $S^{2}$, the YangMills equation for $G$ essentially reproduced the Morse theory picture for the loop space $\Omega G$. Similar ideas can be found in papers concerning magnetic monopoles (see $[4,6,10])$. The purpose of the present paper is to work out this idea exactly and to complete it.

In the first section we investigate a mapping $\Phi: \mathscr{C}(P) \rightarrow \Omega G(\operatorname{see}[4,12])$ from the space of connections on a principal fibre bundle $\left(P, S^{2}, G\right)$ onto a subspace of $\Omega G$ determined by the topological type of $P$.

In Sect. 2 we show that the mapping $\Phi$ carries the critical points of the YangMills functional into the critical points of the energy integral on $\Omega G$. For this we prove that the holonomy group of a Yang-Mills connection over $S^{2}$ is either trivial or the group $S^{1}$. Furthermore, calculating the index and the nullity of a YangMills connection over $S^{2}$ by means of cohomology theory and studying the Jacobi equation on the corresponding closed geodesic, we verify that, after identification 
of gauge equivalent Yang-Mills connections, the index of a critical point of the Yang-Mills functional is equal to the index of the corresponding critical point of the energy integral. Moreover, in both cases the nullity is equal to zero. This means that the equivalence classes of Yang-Mills connections are isolated.

In Sect. 3 we prove an explicit isolation theorem for any Yang-Mills connection over $S^{2}$. In a sense this result corresponds to an isolation phenomenon for Yang-Mills fields over $S^{4}$ announced by Bourguignon and Lawson [3].

\section{A Map from the Space of Gauge Fields over $S^{2}$ Into the Loop-Space of the Structure Group}

Let $\mathscr{C}(P)$ denote the space of smooth connections on a principal fibre bundle $P$. The gauge group $\mathscr{G}(P)$, on $\mathscr{C}(P)$, is the group of automorphisms which act on $P$. Denote by $\mathscr{G}_{0}(P)$ the group of automorphisms preserving a fixed point $p_{0} \in P$.

Now suppose that $\left(P, \pi, S^{2}, G\right)$ is a principal fibre bundle over $S^{2}$, where $G$ is a connected Lie group. Let $\Omega G$ be the loop space of $G$, i.e. the Hilbert manifold of $H^{1}$-maps of the unit circle $S^{1}$ into $G$ taking $1 \in S^{1}$ to the unit of $G$. Then the mapping $\Phi: \mathscr{C}(P) \rightarrow \Omega G$ is defined in the following way. Identify $S^{2}$ with $C \cup\{\infty\}(C$ is the complex plane) and set $V_{1}=S^{2} \backslash\{0\}$ and $V_{2}=S^{2} \backslash\{\infty\}$. Denote by $\tau(Z, \gamma)$ the parallel displacement along the curve $\gamma:[0,1] \rightarrow S^{2}$ by means of the connection $Z \in \mathscr{C}(P)$ and fix a point $p_{0} \in \pi^{-1}(\infty)$. If $\gamma_{x}^{1}$ and $\gamma_{x}^{2}$ are the curves

$$
\begin{gathered}
\gamma_{x}^{1}: t \in[0,1] \rightarrow t^{-1} \cdot x \in S^{2}, \quad x \in V_{1}, \\
\gamma_{x}^{2}: t \in[0,1] \rightarrow t \cdot x \in S^{2}, \quad x \in V_{2} .
\end{gathered}
$$

we define

$$
\begin{array}{ll}
s_{1}^{Z}(x)=\tau\left(Z, \gamma_{x}^{1}\right)\left(p_{0}\right), & x \in V_{1}, \\
s_{2}^{Z}(x)=\tau\left(Z, \gamma_{x}^{2}\right)\left(p_{1}^{Z}\right), & x \in V_{2} .
\end{array}
$$

Here $p_{1}^{Z}=\tau\left(Z, \gamma_{1}\right)\left(p_{0}\right)$, where $\gamma_{1}$ is the curve $t \in[0,1] \rightarrow \frac{1-t}{t} \in S^{2}$.

Proposition 1.1. The mappings $s_{1}^{Z}: V_{1} \rightarrow P$ and $s_{2}^{Z}: V_{2} \rightarrow P$ are smooth sections of the bundle $\left(P, \pi, S^{2}, G\right)$.

Now let $\varrho_{Z}: S^{1} \rightarrow G$ be the mapping which is given by the condition

$$
s_{2}^{Z}(x)=s_{1}^{Z}(x) \cdot \varrho_{Z}(x) \text { for } x \in S^{1},
$$

and define $\Phi(Z)=\varrho_{Z}$.

Proposition 1.2. Let $f \in \mathscr{G}(P)$ and $g \in G$ such that $f\left(p_{0}\right)=p_{0} \cdot g$. Then $\Phi\left(f^{*} Z\right)$ $=g^{-1} \Phi(Z) g$.

Proof. This follows from

$$
f \circ \tau\left(f^{*} Z, \gamma\right)=\tau(Z, \gamma) \circ f \text { for all curves } \gamma .
$$

From Proposition 1.2 we obtain $\Phi\left(f^{*} Z\right)=\Phi(Z)$ for $f \in \mathscr{G}_{0}(P)$. Thus we have a map $\Phi_{0}: \mathscr{C}(P) / \mathscr{G}_{0}(P) \rightarrow \Omega G$ induced by $\Phi$. 
Let us recall that $P$ determines a homotopy class $[P]$ in $\left[S^{1} ; G\right]$, i.e. a connected component of $\Omega G$ (see [13, Sect. 18]). Then we prove:

Proposition 1.3. The image of the map $\Phi_{0}$ is the subspace $[P] \cap \Lambda^{\infty} G$, where $\Lambda^{\infty} G$ is the space of smooth mappings of $S^{1}$ into $G$.

Proof. Let $Z \in \mathscr{C}(P)$. According to Proposition 1.1, $\varrho_{Z} \in \Lambda^{\infty} G$ holds. Moreover, for the charts

$$
\varphi_{i}:(x, g) \in V_{i} \times G \rightarrow s_{i}^{Z}(x) \cdot g \in \pi^{-1}\left(V_{i}\right), \quad i=1,2
$$

of the bundle $P$, we obtain $g_{\left.12\right|_{s^{1}}}=\varrho_{Z}$, where $g_{12}$ is the transition function. Hence, $\varrho_{Z}$ is the characteristic map, and we have $\varrho_{Z} \in[P] \cap \Lambda^{\infty} G$.

Conversely, let $\varrho \in[P] \cap \Lambda^{\infty} G$, and define $\varrho: V_{1} \cap V_{2} \rightarrow G$ by $\varrho(x)=\varrho\left(\frac{x}{|x|}\right)$. From the existence of a principal fibre bundle $\left(Q, S^{2}, G\right)$ with charts $\psi_{i}: V_{i} \times G \rightarrow Q$, $i=1,2$, whose transition function is $\varrho$ (see [14, Proposition II.2.1]) and from the condition $\varrho \in[P]$, implying the equivalence of the bundles $P$ and $Q$, we infer the existence of sections $s_{i}: V_{i} \rightarrow P, i=1,2$, satisfying $s_{1}(\infty)=p_{0}$ and $s_{2}(x)=s_{1}(x) \cdot \bar{\varrho}(x)$ for $x \in V_{1} \cap V_{2}$. Let $\alpha:(0, \infty) \rightarrow[0,1]$ be a smooth mapping with $\alpha(t)=0$ for $t<\varepsilon_{1}$ and $\alpha(t)=1$ for $t>\varepsilon_{2}, 0<\varepsilon_{1}<\varepsilon_{2}$. Using that $\{x, i x\}$ is a basis of the tangent space of $S^{2}=C \cup\{\infty\}$ at $x$ for $x \in V_{1} \cap V_{2}$, we define a 1 -form $Z_{\varrho}^{1}$ on $V_{1}$ by

$$
\begin{gathered}
Z_{\varrho, x}^{1}(x)=0, \quad Z_{\varrho, x}^{1}(i x)=\left.(\alpha(|x|)-1) d L_{\bar{\varrho}(x)} \frac{d}{d t} \bar{\varrho}\left(e^{i t} x\right)^{-1}\right|_{t=0} \text { for } x \in V_{1} \cap V_{2}, \\
Z_{\varrho, \infty}^{1}=0,
\end{gathered}
$$

and a 1-form $Z_{\varrho}^{2}$ on $V_{2}$ by

$$
\begin{gathered}
Z_{\varrho, x}^{2}(x)=0, \quad Z_{\varrho, x}^{2}(i x)=\left.\alpha(|x|) d R_{\bar{\varrho}(x)} \frac{d}{d t} \bar{\varrho}\left(e^{i t} x\right)^{-1}\right|_{t=0} \text { for } x \in V_{1} \cap V_{2}, \\
Z_{\varrho, 0}^{2}=0 .
\end{gathered}
$$

It is clear that the forms $Z_{e}^{1}$ and $Z_{\varrho}^{2}$ are smooth. Moreover, there exists a connection $Z^{\varrho} \in \mathscr{C}(P)$ with $s_{1}^{*} Z^{\varrho}=Z_{\varrho}^{1}$ and $s_{2}^{*} Z^{\varrho}=Z_{\varrho}^{2}$ (see [8, Proposition II.1.4]). Since $s_{1}^{Z^{\varrho}}=s_{1}$ and $s_{2}^{Z^{\varrho}}=s_{2}$, we have $\Phi\left(Z^{\varrho}\right)=\varrho$, and our statement is proved.

We note that the proof of Proposition 1.3 yields the construction of such a mapping $\psi:[P] \cap \Lambda^{\infty} G \rightarrow \mathscr{C}(P) / \mathscr{G}_{0}(P)$ that $\Phi_{0} \circ \psi$ is the identity of $[P] \cap \Lambda^{\infty} G$.

Remark. Fix a connection $Z \in \mathscr{C}(P)$, a point $p_{0} \in \pi^{-1}(\infty)$ and consider a 1-form $\eta$ with values in the adjoint Lie algebra bundle $\operatorname{Ad} P=P \times_{\mathrm{Ad}}$ g. If $\gamma$ is a curve in $S^{2}$ with $\gamma(0)=\infty$, we define the integral

$$
\int_{\gamma}^{(Z)} \eta \in \mathfrak{g}
$$

in the following way: Let $\gamma^{*}$ be the $Z$-horizontal lift of $\gamma$ such that $\gamma^{*}(0)=p_{0}$ and put

$$
\int_{\gamma}^{(\mathrm{Z})} \eta:=\int_{\gamma^{*}} \eta
$$


The values of this integral are elements of the Lie algebra $g$. Now we can easily describe the differential

$$
d \Phi: T_{Z} \mathscr{C}(P)=\Gamma\left(\operatorname{Ad} P \otimes T^{*} M\right) \rightarrow T_{\Phi(Z)} \Omega G
$$

if we identify the tangent space $T_{\Phi(Z)} \Omega G$ with the space of curves in $g$ (using left translations in the group $G) . d \Phi$ is given by

$$
d \Phi(\eta)(\theta)=\int_{\gamma_{\theta}}^{(\mathrm{Z})} \eta
$$

with $\theta \in S^{1} \cdot \gamma_{\theta}$ denotes the closed curve $[0, \infty] \cup\{t \cdot \theta, 0 \leqq t \leqq \infty\}$ in $S^{2}$.

\section{A Correspondence Between Yang-Mills Connections and Closed Geodesics}

Let $G$ be a compact and connected Lie group with the Lie algebra $g$ and a fixed Ad-invariant scalar product on $\mathfrak{g}$. $\operatorname{By} \operatorname{Hom}\left(S^{1}, G\right)$ we denote the set of all homomorphisms $S^{1} \rightarrow G$. We note that sometimes we will identify $\varrho \in \operatorname{Hom}\left(S^{1}, G\right)$ with the closed geodesic $t \in[0,1] \rightarrow \varrho\left(e^{2 \pi i t}\right)$. A pair $(P, Z)$, where $P$ is a $G$-principal fibre bundle over the oriented Riemannian manifold $M$ and $Z$ is a Yang-Mills connection on $P$, we call a Yang-Mills field over $M$ with structure group $G$. The set of all these pairs we denote by $\operatorname{YM}(M ; G)$. We recall that $Z \in \mathscr{C}(P)$ is a Yang-Mills connection if it is a solution of the Yang-Mills equation $D_{Z} * \Omega_{Z}=0$. Here $\Omega_{Z}$ denotes the curvature of $Z$; it is a section of $\operatorname{Ad} P \otimes \Lambda^{2} T^{*} M$, where $\operatorname{Ad} P=P \times_{\operatorname{Ad}} g$ is the vector bundle associated with $P$ by means of the adjoint representation. The operator $*$ is the Hodge operator and $D_{Z}$ is the covariant differential defined by $Z$. Sometimes we will interpret a section $\xi \in \Gamma(\operatorname{Ad} P)$ as an equivariant mapping $P \rightarrow \mathfrak{g}$.

In this section we investigate the relation between Yang-Mills connections over $S^{2}$ and closed geodesics. First we define a mapping $\theta: \operatorname{Hom}\left(S^{1}, G\right)$ $\rightarrow \mathrm{YM}\left(S^{2}, G\right)$. For this we consider the Hopf bundle $\left(S^{3}, S^{2}, S^{1}\right)$, where $S^{1}$ acts on

$$
S^{3}=\left\{\left(z_{1}, z_{2}\right) \in C^{2} ;\left|z_{1}\right|^{2}+\left|z_{2}\right|^{2}=1\right\}
$$

by $\left(z_{1}, z_{2}\right) \cdot z=\left(z_{1} \cdot z, z_{2} \cdot z\right)$. Then

$$
A=\frac{1}{2}\left(\bar{z}_{1} d z_{1}-z_{1} d \bar{z}_{1}+\bar{z}_{2} d z_{2}-z_{2} d \bar{z}_{2}\right)
$$

is an irreducible Yang-Mills connection on $S^{3}$ with the curvature $\Omega_{A}=\frac{2 \pi i}{\operatorname{vol}\left(S^{2}\right)} \otimes d S^{2}$. Let $\varrho \in \operatorname{Hom}\left(S^{1}, G\right)$. The associated bundle $P_{\varrho}=S^{3} \times{ }_{\varrho} G$ is a $G$-principal fibre bundle, where $G$ acts on $P_{e}$ by $[u, g] \cdot g_{1}=\left[u, g g_{1}\right]$. It is clear that $i_{\varrho}: u \in S^{3} \rightarrow[u, e] \in P_{\varrho}$ is a homomorphism of fibre bundles with the homomorphism $\varrho: S^{1} \rightarrow G$. Consequently, $i_{\varrho}$ induces a connection $A_{\varrho}$ on $P_{\varrho}$, determined by $i_{\varrho}^{*} A_{\varrho}$ $=\varrho_{*} A$. Since $i_{e}^{*} \Omega_{A_{e}}=\varrho_{*} \Omega_{A}$, we can easily verify that $A_{\varrho}$ is a Yang-Mills connection on $P_{\varrho}$. Thus, setting $\theta(\varrho)=\left(P_{\varrho}, A_{\varrho}\right)$, the mapping $\theta: \operatorname{Hom}\left(S^{1}, G\right) \rightarrow \mathrm{YM}\left(S^{2}, G\right)$ is correctly defined.

Proposition 2.1. Let $\varrho$ and $\varrho$ be in $\operatorname{Hom}\left(S^{1}, G\right)$. Then the Yang-Mills fields $\left(P_{\varrho}, A_{\varrho}\right)$ and $\left(P_{\bar{\varrho}}, A_{\bar{Q}}\right)$ are equivalent, i.e. there exists a gauge transformation $f: P_{\varrho} \rightarrow P_{\bar{Q}}$ with $f^{*} A_{\bar{\varrho}}=A_{\varrho}$ if and only if the homomorphisms $\varrho$ and $\varrho$ are conjugate. 
Proof. Suppose $\varrho=g_{1} \bar{\varrho} g_{1}^{-1}, g_{1} \in G$, and denote by $[,]_{\varrho}$ and $[,]_{\bar{\varrho}}$, respectively, the equivalence classes in $P_{\varrho}$ and $P_{\bar{\varrho}}$. Then the gauge transformation $f:[u, g]_{\varrho} \in P_{\varrho}$ $\rightarrow\left[u, g_{1} g\right]_{\bar{\varrho}} \in P_{\bar{\varrho}}$ satisfies $i_{\varrho}^{*} f^{*} A_{\bar{\varrho}}=\varrho_{*} A$. This implies $f^{*} A_{\bar{\varrho}}=A_{\varrho}$, since $A_{\varrho}$ is determined by $i_{\varrho}^{*} A_{\varrho}=\varrho_{*} A$.

Conversely, let $f: P_{\varrho} \rightarrow P_{\bar{\varrho}}$ be a gauge transformation with $f^{*} A_{\varrho}=A_{\varrho}$. We define a map $\bar{f}: S^{3} \rightarrow G$ by the condition $f \circ i_{\varrho}(u)=i_{\bar{\varrho}}(u) \cdot \bar{f}(u)$ for $u \in S^{3}$ and observe $\bar{f}(u) \varrho(z) \bar{f}(u z)^{-1}=\varrho(z)$ for $u \in S^{3}$ and $z \in S^{1}$.

In order to complete the proof we have to verify that $\bar{f}$ is constant. The definition of $\bar{f}$ yields

$$
\begin{aligned}
\varrho_{*} A & =i_{\varrho}^{*} A_{\varrho}=i_{\varrho}^{*} f^{*} A_{\bar{\varrho}}=\left(R_{\bar{f}} \circ i_{\bar{\varrho}}\right) * A_{\bar{\varrho}}=\operatorname{Ad}\left(\bar{f}^{-1}\right)\left(i_{\bar{\varrho}}^{*} A_{\bar{\varrho}}\right)+\bar{f}^{*} \omega \\
& =\operatorname{Ad}\left(\bar{f}^{-1}\right)\left(\bar{\varrho}_{*} A\right)+\bar{f}^{*} \omega,
\end{aligned}
$$

where $\omega$ is the Maurer-Cartan form of $G$. Hence, if $t \in T S^{3}$ is horizontal with respect to the connection $A$, then $\bar{f}^{*} \omega(t)=0$ holds. Consequently, $\bar{f}$ is constant along any horizontal curve in $S^{3}$. Since $A$ is irreducible, $\bar{f}$ is constant on $S^{3}$.

From Proposition 2.1, we obtain that $\theta$ induces an injective mapping $\bar{\theta}: \overline{\operatorname{Hom}}\left(S^{1}, G\right) \rightarrow \overline{\mathrm{YM}}\left(S^{2}, G\right)$ from the set $\overline{\operatorname{Hom}}\left(S^{1}, G\right)$ of conjugacy classes of homomorphisms $S^{1} \rightarrow G$ into the set $\overline{\mathrm{YM}}\left(S^{2}, G\right)$ of equivalence classes in $\operatorname{YM}\left(S^{2}, G\right)$. Before we show that the mapping $\bar{\theta}$ is also surjective, we prove:

Proposition 2.2. Let $\left(P, M^{2}, G\right)$ be a principal fibre bundle, where $M^{2}$ is a connected Riemann surface, and let $Z$ be a Yang-Mills connection on $P$. Then the holonomy group of $Z$ is discrete or one-dimensional. In particular, if $M^{2}=S^{2}$, then the holonomy group is either trivial or $S^{1}$.

Proof. Let $\Phi\left(p_{0}\right)$ be the holonomy group and $P\left(p_{0}\right)$ be the holonomy bundle of $Z$ with reference point $p_{0} \in P$. Because of $D_{Z} * \Omega_{Z}=0$ the mapping $* \Omega_{Z}: P \rightarrow \mathrm{g}$ is constant along horizontal curves. Hence, $* \Omega_{Z}$ is equal to $X \in \mathfrak{g}$ on $P\left(p_{0}\right)$. Applying the holonomy theorem of Ambrose and Singer (see [8, Theorem II.8.1]), we obtain that the Lie algebra of $\Phi\left(p_{0}\right)$ is spanned by $X$. Therefore $\Phi\left(p_{0}\right)$ is discrete or onedimensional.

Suppose $M^{2}=S^{2}$. Then $\Phi\left(p_{0}\right)$ is connected (see [8, Sect. II.4]). Consequently, $\Phi\left(p_{0}\right)$ is trivial, the circle $S^{1}$ or the line $R$. Suppose $\Phi\left(p_{0}\right)=R$. Since $R$ is simply connected, the holonomy bundle $\left(P\left(p_{0}\right), S^{2}, \Phi\left(p_{0}\right)\right)$ is trivial. Let $\hat{Z} \in \mathscr{C}\left(P\left(p_{0}\right)\right)$ be the reduced Yang-Mills connection. Because $\Phi\left(p_{0}\right)$ is an abelian group, the YangMills equation $D_{\hat{Z}} * \Omega_{\hat{Z}}=0$ is equivalent to $d *\left(s^{*} \Omega_{\hat{Z}}\right)=0$, where $s: S^{2} \rightarrow P\left(p_{0}\right)$ is a global section. Hence $s^{*} \Omega_{\hat{Z}}=c \cdot d S^{2}, c \in R$. Because of $s^{*} \Omega_{\hat{Z}}=s^{*} d \hat{Z}=d\left(s^{*} \hat{Z}\right)$ we have $d\left(s^{*} \hat{Z}\right)=c \cdot d S^{2}$. By integration we obtain $0=c \cdot \operatorname{vol}\left(S^{2}\right)$. Consequently, $\Omega_{\hat{Z}}$ vanishes. Since this contradicts the irreducibility of $\hat{Z}$, the proposition is proved.

Proposition 2.3. Let $(P, Z) \in \mathrm{YM}\left(S^{2}, G\right)$ and let $X=* \Omega_{\mathrm{Z}}\left(p_{0}\right), p_{0} \in P$. Then $\varrho: t \in[0,1] \rightarrow \exp \left(t \cdot X \cdot \operatorname{vol}\left(S^{2}\right)\right) \in G$ is a closed geodesic, and the Yang-Mills fields $(P, Z)$ and $\left(P_{\varrho}, A_{\varrho}\right)$ are equivalent.

Proof. In the case $G=S^{1}$ we have $\Omega_{Z}=X \otimes d S^{2}, X \in i R$. Then the first Chern class of the bundle $P$ is $c_{1}(P)=-\frac{X}{2 \pi i} \operatorname{vol}\left(S^{2}\right)$. Since $c_{1}(P)=k$ is an integer, it is obvious that

$$
\varrho: t \in[0,1] \rightarrow e^{t \cdot X \cdot \operatorname{vol}\left(S^{2}\right)} \in S^{1}
$$


is a closed geodesic. Further, for the Yang-Mills field $\left(P_{\varrho}, A_{\varrho}\right)$ we have

$$
\Omega_{A_{Q}}=\varrho_{*} \Omega_{A}=\varrho_{*}\left(\frac{2 \pi i}{\operatorname{vol}\left(S^{2}\right)}\right) \otimes d S^{2}=X \otimes d S^{2}=\Omega_{Z} .
$$

Hence $c_{1}(P)=c_{1}\left(P_{e}\right)$. It follows that there exists a gauge transformation $f_{1}: P_{e} \rightarrow P$. Since $\Omega_{f_{1}^{*} Z}=\Omega_{Z}$, interpreted as 2-forms on $S^{2}$, and since the Picard group of $S^{2}$ is trivial, we obtain from the Theorem of Weil (see [9]) that there exists a gauge transformation $f_{2}: P_{\varrho} \rightarrow P_{\varrho}$ satisfying $f_{2}^{*} f_{1}^{*} Z=A_{\varrho}$. Thus our statement is true for $G=S^{1}$.

Now let $G$ be any compact and connected Lie group. The connection $Z \in \mathscr{C}(P)$ reduces to a connection $\hat{Z}$ on the holonomy bundle $\left(P\left(p_{0}\right), S^{2}, \Phi\left(p_{0}\right)\right)$. By Proposition $2.2 \Phi\left(p_{0}\right)$ is either trivial or $S^{1}$. Therefore, we have already shown that

$$
\hat{\varrho}: t \in[0,1] \rightarrow \exp \left(t \cdot X \cdot \operatorname{vol}\left(S^{2}\right)\right) \in \Phi\left(p_{0}\right)
$$

is a closed geodesic in $\Phi\left(p_{0}\right)$ and so is the embedded curve

$$
\varrho: t \in[0,1] \rightarrow \exp \left(t \cdot X \cdot \operatorname{vol}\left(S^{2}\right)\right) \in G
$$

in $G$. Moreover, there is a gauge transformation $\hat{f}: P_{\hat{Q}} \rightarrow P\left(p_{0}\right)$ with $\hat{f}^{*} \hat{Z}=A_{\hat{Q}}$. Extending $\hat{f}$ to a gauge transformation $P_{Q} \rightarrow P$, we see the equivalence of $(P, Z)$ and $\left(P_{\varrho}, A_{\varrho}\right)$.

So we have proved:

Theorem 2.1. The mapping $\bar{\theta}: \overline{\mathrm{Hom}}\left(S^{1}, G\right) \rightarrow \overline{\mathrm{YM}}\left(S^{2}, G\right)$ is a bijective correspondence between conjugacy classes of homomorphisms $S^{1} \rightarrow G$ and equivalence classes of Yang-Mills fields over $S^{2}$ with structure group $G$.

We remark that a simple calculation leads to $E(\varrho)=\operatorname{vol}\left(S^{2}\right) L\left(A_{\varrho}\right)$ for $\varrho \in \operatorname{Hom}\left(S^{1}, G\right)$.

The following corollary shows that the correspondence between $\overline{\operatorname{Hom}}\left(S^{1}, G\right)$ and $\overline{\mathrm{YM}}\left(S^{2}, G\right)$ is realized by the mapping $\Phi: Z \in \mathscr{C}(P) \rightarrow \varrho_{Z} \in \Omega G$.

Corollary 2.1. Let $(P, Z) \in \mathrm{YM}\left(S^{2}, G\right)$. Then $\varrho_{Z} \in \operatorname{Hom}\left(S^{1}, G\right)$ and the Yang-Mills fields $(P, Z)$ and $\left(P_{e Z}, A_{\varrho Z}\right)$ are equivalent.

Proof. Consider the Hopf bundle with the fixed connection $A$ and set $p_{0}=(1,0)$. Then we see that the sections $s_{1}^{A}$ and $s_{2}^{A}$ are given by

$$
\begin{gathered}
s_{1}^{A}(x)=\left(|x|\left(1+|x|^{2}\right)^{-1 / 2}, x^{-1}|x|\left(1+|x|^{2}\right)^{-1 / 2}\right), \quad x \in V_{1}, \\
s_{2}^{A}(x)=\left(x\left(1+|x|^{2}\right)^{-1 / 2},\left(1+|x|^{2}\right)^{-1 / 2}\right), \quad x \in V_{2} .
\end{gathered}
$$

Consequently $\varrho_{A}=\mathrm{id}_{S^{1}}$. Now, applying Theorem 2.1, the proof is straightforward.

Let us recall that the Yang-Mills connections on a principal fibre bundle $P$ are the critical points of the Yang-Mills functional $L: \mathscr{C}(P) \rightarrow R$. Since this functional is constant on the orbits of the action of the gauge group $\mathscr{G}(P)$, we have mappings $L: \mathscr{C}(P) / \mathscr{G}(P) \rightarrow R$ and $L: \mathscr{C}(P) / \mathscr{G}_{0}(P) \rightarrow R$. Now we will calculate the index and the nullity of a Yang-Mills connection over $S^{2}$, understood as a critical point of the functional $L: \mathscr{C}(P) / \mathscr{G}(P) \rightarrow R$. For definitions we refer to [2].

Let $\varrho \in \operatorname{Hom}\left(S^{1}, G\right), X=\left.\frac{d}{d t} \varrho\left(e^{2 \pi i t}\right)\right|_{t=0}$ and choose a maximal abelian subalgebra $t$ of the Lie algebra $\mathrm{g}$ of $G$ with $X \in t$. It is well-known (see [1]) that there 
exists a basis $e_{1}, \ldots, e_{n}$ of $\mathfrak{g}$ satisfying

$$
\begin{gathered}
\operatorname{ad}(X)\left(e_{2 k-1}\right)=2 \pi \theta_{k}(X) e_{2 k}, \operatorname{ad}(X)\left(e_{2 k}\right)=-2 \pi \theta_{k}(X) e_{2 k-1}, \quad k=1, \ldots, l, \\
\operatorname{ad}(X)\left(e_{s}\right)=0, \quad s=2 l+1, \ldots, n,
\end{gathered}
$$

where the linear forms $\theta_{k}: t \rightarrow R, k=1, \ldots, l$, are the roots of $G$.

Proposition 2.4. For the Yang-Mills connection $A_{\varrho} \in \mathscr{C}\left(P_{\varrho}\right)$, we have

$$
\operatorname{Index}\left(A_{\varrho}\right)=2 \sum_{\substack{k=1 \\ \theta_{k}(X) \neq 0}}^{l}\left(\left|\theta_{k}(X)\right|-1\right) \text { and } \operatorname{Nullity}\left(A_{\varrho}\right)=0 \text {. }
$$

For the proof we use a fact concerning the index and the nullity of a Yang-Mills field $(P, Z)$ over a compact Riemann surface $M^{2}$ with structure group $G$. Endow the bundle $\operatorname{Ad} P \otimes C=P \times{ }_{\text {Ad }}(g \otimes C)$ with the complex structure defined by $Z$ and consider the holomorphic bundle morphism

$$
\Lambda_{Z}: \varphi \in \operatorname{Ad} P \otimes C \rightarrow i\left[* \Omega_{Z}, \varphi\right] \in \operatorname{Ad} P \otimes C,
$$

where $[$,$] is the Lie bracket. Let P^{\lambda}=\left\{\varphi \in \operatorname{Ad} P \otimes C ; \Lambda_{Z}(\varphi)=\lambda \cdot \varphi\right\}$ and $P^{+}=\bigoplus_{\lambda>0} P^{\lambda}$ (one easily sees that $P^{\lambda}$ is a holomorphic subbundle of $\operatorname{Ad} P \otimes C$, and so is $P^{+}$). Then the following proposition holds:

Proposition 2.5. If $M^{2}$ is a surface of genus $g$, then

$\operatorname{Index}(Z)=2\left\{c_{1}\left(P^{+}\right)+\operatorname{dim}_{c}\left(P^{+}\right)(g-1)\right\} \quad$ and $\operatorname{Nullity}(Z)=2 \operatorname{dim}_{c} H^{1}\left(M^{2} ; P^{0}\right)$.

Here $H^{1}\left(M^{2} ; P^{0}\right)$ is the first cohomology group of $M^{2}$ with coefficients in the sheaf of holomorphic sections of $P^{0}$.

The proof of this proposition is given in [2].

Proof of Proposition 2.4. Fix $X=\left.\frac{d}{d t} \varrho\left(e^{2 \pi i t}\right)\right|_{t=0}$, and let

$$
\mathfrak{g}^{\lambda}=\left\{Y \in \mathfrak{g} \otimes C ; \frac{i}{\operatorname{vol}\left(S^{2}\right)} \operatorname{ad}(X)(Y)=\lambda \cdot Y\right\} \text {. }
$$

Because of $* \Omega_{A_{\varrho}}\left(i_{\varrho}(u)\right)=\frac{X}{\operatorname{vol}\left(S^{2}\right)}$ for $u \in S^{3}$, we see that the holomorphic bundle $P_{\varrho}^{\lambda}$ and the bundle $S^{3} \times{ }_{\text {Adoe }} \mathfrak{g}^{\lambda}$ endowed with the complex structure defined by the connection $A$ on $S^{3}$ are isomorphic (consider the mapping

$$
\left.[u, Y] \in S^{3} \times_{\mathrm{Ad} \varrho \varrho} \mathrm{g}^{\lambda} \rightarrow[[u, e], Y] \in P_{\varrho}^{\lambda} \subseteq P_{\varrho} \times_{\mathrm{Ad}}(\mathfrak{g} \otimes C)\right) .
$$

Arrange the basis $e_{1}, \ldots, e_{n}$ of $g$ so that $\theta_{k}(X)>0$ for $k=1, \ldots, m$ and $\theta_{k}(X)=0$ for $m<k \leqq l$, and let

$$
f_{k}=\frac{e_{2 k-1}+i e_{2 k}}{\sqrt{2}} \text { and } h_{k}=\frac{e_{2 k-1}-i e_{2 k}}{\sqrt{2}} \text { for } k=1, \ldots, m .
$$


Then $f_{1}, \ldots, f_{m}, h_{1}, \ldots, h_{m}, e_{2 m+1}, \ldots, e_{n}$ is a basis of $\mathfrak{g} \otimes C$ satisfying

$$
\begin{gathered}
\frac{i}{\operatorname{vol}\left(S^{2}\right)} \operatorname{ad}(X)\left(f_{k}\right)=\frac{2 \pi \theta_{k}(X)}{\operatorname{vol}\left(S^{2}\right)} f_{k}, \quad k=1, \ldots, m, \\
\frac{i}{\operatorname{vol}\left(S^{2}\right)} \operatorname{ad}(X)\left(h_{k}\right)=-\frac{2 \pi \theta_{k}(X)}{\operatorname{vol}\left(S^{2}\right)} h_{k}, \quad k=1, \ldots, m, \\
\frac{i}{\operatorname{vol}\left(S^{2}\right)} \operatorname{ad}(X)\left(e_{s}\right)=0, \quad s=2 m+1, \ldots, n .
\end{gathered}
$$

Consequently, the bundles

$$
P_{\varrho}^{+}=\bigoplus_{\lambda>0}\left(S^{3} \times{ }_{\operatorname{Ad} \circ \varrho} g^{\lambda}\right) \text { and } \bigoplus_{k=1}^{m}\left(S^{3} \times{ }_{\operatorname{Ad} \circ \varrho} C \cdot f_{k}\right)
$$

are topologically isomorphic. Moreover calculating the curvature of the covariant derivative in $S^{3} \times{ }_{\text {Ad } \varrho} C \cdot f_{k}$ defined by $A \in \mathscr{C}\left(S^{3}\right)$, we obtain that the first Chern class of $S^{3} \times{ }_{\text {Ad } \varrho} C \cdot f_{k}$ is $\theta_{k}(X)$. Applying Proposition 2.5, we have

$\operatorname{Index}\left(A_{\varrho}\right)=2\left\{c_{1}\left(P_{\varrho}^{+}\right)-\operatorname{dim}_{c}\left(P_{\varrho}^{+}\right)\right\}=2\left\{\left(\sum_{k=1}^{m} \theta_{k}(X)\right)-m\right\}=2 \sum_{\substack{k=1 \\ \theta_{k}(X) \neq 0}}^{l}\left(\left|\theta_{k}(X)\right|-1\right)$.

Further, we see that $\mathrm{g}^{0}$ is spanned by the vectors $e_{s}, s=2 m+1, \ldots, n$. Since $\operatorname{ad}(X)$ vanishes on $g^{0}$, the representation $A d \circ \varrho: S^{1} \rightarrow G L\left(g^{0}\right)$ is trivial. Therefore, $x \in S^{2}$ $\rightarrow\left[u, e_{s}\right] \in S^{3} \times{ }_{\text {Ad } \varrho} g^{0}$, where $u$ is in the fibre over $x$, is a holomorphic section. Because of $H^{1}\left(S^{2} ; C\right)=0$ (see [5], Theorem 12.7) we arrive at

$$
\operatorname{Nullity}\left(A_{\varrho}\right)=2 \operatorname{dim}_{c} H^{1}\left(S^{2} ; P^{0}\right)=2 \operatorname{dim}_{c}\left\{\bigoplus_{s=2 m+1}^{n} H^{1}\left(S^{2} ; C\right)\right\}=0 .
$$

This concludes the proof.

If a Yang-Mills connection on $P$ is considered as a critical point of the functional $L: \mathscr{C}(P) / \mathscr{G}_{0}(P) \rightarrow R$, it is natural to define the index and the nullity of $Z$ as follows:

$$
\begin{gathered}
\operatorname{Index}_{0}(Z)=\operatorname{Index}(Z), \\
\operatorname{Nullity}_{0}(Z)=\operatorname{Nullity}(Z)+\operatorname{dim}\left(D_{z}(\Gamma(\operatorname{Ad} P)) / D_{Z}\left(\Gamma_{0}(\operatorname{Ad} P)\right)\right) .
\end{gathered}
$$

Here $\Gamma_{0}(\operatorname{Ad} P)=\left\{\xi \in \Gamma(\operatorname{Ad} P) ; \xi\left(p_{0}\right)=0\right\}$ is the Lie algebra of $\mathscr{G}_{0}(P)$.

Proposition 2.6. For the Yang-Mills connection $A_{\varrho} \in \mathscr{C}\left(P_{\varrho}\right)$ we have Nullity $_{0}\left(A_{\varrho}\right)=\operatorname{dimg}-\operatorname{dimg}_{X}$, where $\mathfrak{g}_{X}=\{Y \in \mathfrak{g} ;[X, Y]=0\}$.

Proof. Since the sequence

$$
0 \rightarrow \operatorname{ker} D_{A_{\varrho}} \rightarrow \Gamma\left(\operatorname{Ad} P_{\varrho}\right) / \Gamma_{0}\left(\operatorname{Ad} P_{\varrho}\right) \stackrel{D_{A_{\varrho}}}{\longrightarrow} D_{A_{\varrho}}\left(\Gamma\left(\operatorname{Ad} P_{\varrho}\right)\right) / D_{A_{\varrho}}\left(\Gamma_{0}(\operatorname{Ad} P)\right) \rightarrow 0
$$

is exact and since $\Gamma\left(\operatorname{Ad} P_{\varrho}\right) / \Gamma_{0}\left(\operatorname{Ad} P_{\varrho}\right)$ is isomorphic to $\mathfrak{g}$, we have

$$
\operatorname{dim}\left(D_{A_{\varrho}}\left(\Gamma\left(\operatorname{Ad} P_{\varrho}\right)\right) / D_{A_{\varrho}}\left(\Gamma_{0}\left(\operatorname{Ad} P_{\varrho}\right)\right)\right)=\operatorname{dimg}-\operatorname{dim} \operatorname{Ker} D_{A_{\varrho}} .
$$

Hence, using Nullity $\left(A_{\varrho}\right)=0$, it suffices to show that $\operatorname{Ker} D_{A_{\varrho}}$ is isomorphic to $\mathfrak{g}_{X}$. Let $\xi \in \operatorname{Ker} D_{A_{Q}}$ and fix $u_{0} \in S^{3}$. From $D_{A_{Q}} D_{A_{Q}} \xi=\left[\Omega_{A_{Q}}, \xi\right]=0$ we obtain 
$\left[X, \xi\left(i_{\varrho}\left(u_{0}\right)\right)\right]=0$. So we get the mapping $\xi \in \operatorname{Ker} D_{A} \rightarrow \xi\left(i\left(u_{0}\right)\right) \in \mathfrak{g}_{X}$. It is easy to check that this mapping is an isomorphism.

Endow the group $G$ with the biinvariant metric $g$, induced by the Ad-invariant scalar product on $\mathrm{g}$. Then it is well-known that $\operatorname{Hom}\left(S^{1}, G\right)$ is the set of the critical points of the energy integral on $\Omega G$. Therefore, the Morse theory defines the index and the nullity of a closed geodesic $\varrho \in \operatorname{Hom}\left(S^{1}, G\right)$.

Theorem 2.2. Let $\varrho \in \operatorname{Hom}\left(S^{1}, G\right)$ and let $\left(P_{\varrho}, A_{\varrho}\right)$ be the corresponding Yang-Mills field. Then $\operatorname{Index}{ }_{0}\left(A_{\varrho}\right)=\operatorname{Index}(\varrho)$ and $\operatorname{Nullity}_{0}\left(A_{\varrho}\right)=\operatorname{Nullity}(\varrho)$.

Proof. We have to calculate the index and the nullity of the closed geodesic $\varrho$. For this we make use of a relation between index and nullity of a geodesic and the Jacobi equation:

Proposition 2.7. Let $\varrho:[0,1] \rightarrow M$ be a geodesic on the Riemannian manifold $M$ and denote by $J_{\varrho}^{t_{0}}, 0<t_{0} \leqq 1$, the space of Jacobi fields $J:[0,1] \rightarrow T M$ along $\varrho$, satisfying $J(0)=J\left(t_{0}\right)=0$. Then Nullity $(\varrho)=\operatorname{dim} J_{\varrho}^{1}$ and

$$
\operatorname{Index}(\varrho)=\sum_{0<t_{0}<1} \operatorname{dim} J_{\varrho}^{t_{0}} .
$$

For the proof see $[7,2.5 .6,2.5 .9]$.

We want to apply Proposition 2.7 to the closed geodesic

$$
\varrho: t \in[0,1] \rightarrow \exp t \cdot X \in G, X \in \mathfrak{g} .
$$

The following lemma gives a useful characterization of Jacobi fields along $\varrho$.

Lemma 2.1. A vector field $J$ along $\varrho$ is a Jacobi field if and only if the curve $Y: t \in[0,1] \rightarrow d L_{\varrho(t)}^{-1} J(t) \in \mathfrak{g}$ is a solution of the differential equation

$$
\frac{d^{2}}{d t^{2}} Y(t)+\left[X, \frac{d}{d t} Y(t)\right]=0
$$

Now we calculate the dimension of the space of solutions of the problem

$$
\frac{d^{2}}{d t^{2}} Y(t)+\left[X, \frac{d}{d t} Y(t)\right]=0, \quad Y(0)=Y\left(t_{0}\right)=0
$$

for $0<t_{0} \leqq 1$. Fix a basis $e_{1}, \ldots, e_{n}$ of $\mathfrak{g}$ as in the proof of Proposition 2.4. It follows that

$$
Y(t)=\sum_{k=1}^{m}\left(A_{k}(t) e_{2 k-1}+B_{k}(t) e_{2 k}\right)+\sum_{k=2 m+1}^{n} C_{k}(t) e_{k}
$$

is a solution of (1) if and only if $\left(A_{1}, B_{1}, \ldots, A_{m}, B_{m}\right)$ is a solution of

$$
\begin{gathered}
\frac{d^{2}}{d t^{2}} A_{k}(t)-2 \pi \theta_{k}(X) \frac{d}{d t} B_{k}(t)=0, \\
\frac{d^{2}}{d t^{2}} B_{k}(t)+2 \pi \theta_{k}(X) \frac{d}{d t} A_{k}(t)=0, \\
A_{k}(0)=B_{k}(0)=A_{k}\left(t_{0}\right)=B_{k}\left(t_{0}\right)=0, \quad k=1, \ldots, m,
\end{gathered}
$$


and $\left(C_{2 m+1}, \ldots, C_{n}\right)$ is a solution of

$$
\begin{gathered}
\frac{d^{2}}{d t^{2}} C_{k}(t)=0, \\
C_{k}(0)=C_{k}\left(t_{0}\right)=0, \quad k=2 m+1, \ldots, n .
\end{gathered}
$$

From (3) we conclude that $C_{k}, k=2 m+1, \ldots, n$, vanishes. Setting $y_{k}=A_{k}+i B_{k}$ for $k=1, \ldots, m$, problem (2) is equivalent to

$$
\frac{d^{2}}{d t^{2}} y_{k}(t)+i 2 \pi \theta_{k}(X) \frac{d}{d t} y_{k}(t)=0, \quad y_{k}(0)=y_{k}\left(t_{0}\right)=0, \quad k=1, \ldots, m .
$$

The solutions of (4) are given by

$$
\begin{gathered}
y_{k}(t)=z_{k}-z_{k} \cdot e^{-2 \pi i \theta_{k}(X) t}, \quad z_{k} \in C, \quad \text { if } \theta_{k}(X) \cdot t_{0} \in Z, \\
y_{k}=0, \quad \text { otherwise. }
\end{gathered}
$$

Therefore, using $\theta_{k}(X) \in Z, k=1, \ldots, m$, and the fact that $g_{x}$ is spanned by the vectors $e_{2 m+1}, \ldots, e_{n}$, Proposition 2.7 leads to Nullity $(\varrho)=2 m=\operatorname{dimg}-\operatorname{dim} \mathfrak{g}_{X}$ and

$$
\operatorname{Index}(\varrho)=2 \sum_{k=1}^{m}\left(\theta_{k}(X)-1\right) .
$$

This concludes the proof of Theorem 2.2.

It is clear that the energy integral induces a functional $\bar{E}: \overline{\Omega G} \rightarrow R$ on the space $\overline{\Omega G}$ of conjugacy classes of loops. Therefore, a closed geodesic $\varrho \in \operatorname{Hom}\left(S^{1}, G\right)$ can also be considered as a critical point of $\bar{E}$.

We denote the index and the nullity of $\varrho$ by $\operatorname{Index}_{*}(\varrho)$ and Nullity $_{*}(\varrho)$.

Lemma 2.2. Let $\varrho \in \operatorname{Hom}\left(S^{1}, G\right)$. Then Nullity $_{*}(\varrho)=0$.

Proof. It suffices to check that the tangent space $T_{\varrho}[\varrho]$ of the conjugacy class of $\varrho$ at the point $\varrho$ is equal to the space $J_{\varrho}^{1}$ of Jacobi fields $J$ along $\varrho$ satisfying $J(0)$ $=J(1)=0$. The relation $T_{\varrho}[\varrho] \subseteq J_{\varrho}^{1}$ is well-known (see [7, 1.12.4]). To see that $J_{\varrho}^{1} \subseteq T_{\varrho}[\varrho]$ we consider the variations

$$
\varphi_{k}:(s, t) \in R \times[0,1] \rightarrow \exp \left(s \cdot e_{k}\right) \varrho(t) \exp \left(-s \cdot e_{k}\right), \quad k=1, \ldots, 2 m .
$$

where $e_{1}, \ldots, e_{n}$ is the fixed basis of $\mathfrak{g}$. Since the vector fields

$$
\left.\frac{d}{d s} \varphi_{k}(s, t)\right|_{s=0}=d L_{\varrho(t)}\left(e_{k}-\operatorname{Ad}\left(\varrho(t)^{-1}\right) e_{k}\right)
$$

form a basis of $J_{\varrho}^{1}$, the desired result follows.

As an immediate consequence we obtain:

Corollary 2.2. Let $\varrho \in \operatorname{Hom}\left(S^{1}, G\right)$ and let $\left(P_{\varrho}, A_{\varrho}\right)$ be the corresponding Yang-Mills field. Then $\operatorname{Index}\left(A_{\varrho}\right)=\operatorname{Index}_{*}(\varrho)$ and $\operatorname{Nullity}\left(A_{\varrho}\right)=\operatorname{Nullity}_{*}(\varrho)$.

\section{An Isolation Theorem for Yang-Mills Fields over $\boldsymbol{S}^{\mathbf{2}}$}

We consider a $G$-principal fibre bundle $P$ over $S^{2}$ and endow the space $\mathscr{C}(P)$ with the Sobolev topology $H^{1}$, which is defined by a $H^{1}$-Norm \|\|$_{1}$ in $\Gamma(\operatorname{Ad} P)$. For 
details see [11]. We endow the space $\mathscr{C}(P) / \mathscr{G}(P)$ with the quotient space topology. In this section, we will construct for any equivalence class of Yang-Mills connections on $P$ an explicit neighbourhood in which there are no other equivalence classes of Yang-Mills connections. To describe this neighbourhood we will use the mapping dist: $\mathscr{C}(P) \times \mathscr{C}(P) \rightarrow R$ defined by

$$
\operatorname{dist}\left(Z_{1}, Z_{2}\right)=\left(\inf _{f_{1}, f_{2} \in \mathscr{G}(P)} \int_{S^{2}}\left\|\Omega_{f_{1}^{*} Z_{1}}-\Omega_{f_{2}^{*} Z_{2}}\right\|^{2} d S^{2}\right)^{1 / 2} .
$$

The following lemma relates the mapping dist to the topology of $\mathscr{C}(P)$.

Lemma 3.1. Let $Z_{0} \in \mathscr{C}(P)$ and let $\varepsilon$ be a positive real number. Then

$$
\left\{Z \in \mathscr{C}(P) ; \operatorname{dist}\left(Z_{0}, Z\right)<\varepsilon\right\}
$$

is an open subset of $\mathscr{C}(P)$.

Proof. By standard estimates we can show that for any $Z \in \mathscr{C}(P)$ there exists a positive real $c_{Z}$ such that $\operatorname{dist}(Z, Z+\eta) \leqq c_{Z}\|\eta\|_{1}$ for all $\eta \in \Gamma\left(\operatorname{Ad} P \otimes T^{*} M\right)$ with $\|\eta\|_{1} \leqq 1$. Since dist obviously satisfies the triangle inequality, the lemma follows.

Next we describe a correspondence which will be used decisively. Consider a field $(P, Z) \in \mathrm{YM}\left(S^{2}, G\right)$ and interpret $* \Omega_{Z}$ as a mapping $P \rightarrow \mathrm{g}$ satisfying $* \Omega_{\mathrm{Z}}(p \cdot g)$ $=\operatorname{Ad}\left(g^{-1}\right) * \Omega_{Z}(p)$ for all $p \in P$ and all $g \in G$. Since the Yang-Mills equation $D_{Z} * \Omega_{Z}=0$ implies that $* \Omega_{Z}$ is constant along horizontal curves, the image $\mathcal{O}_{Z}$ of the mapping $* \Omega_{Z}$ is an orbit of the adjoint representation. Let $\operatorname{Orb}(G)$ denote the set of all orbits of the adjoint representation and denote by $\operatorname{Orb}^{1}(G)$ the set of the orbits $\mathcal{O} \in \operatorname{Orb}(G)$ with the property that $X \in \mathcal{O}$ leads to $\exp \left(X \cdot \operatorname{vol}\left(S^{2}\right)\right)=1$. By Proposition 2.3 we have $\mathcal{O}_{Z} \in \operatorname{Orb}^{1}(G)$.

Proposition 3.1. The mapping $(P, Z) \in \mathrm{YM}\left(S^{2}, G\right) \rightarrow \mathcal{O}_{Z} \in \operatorname{Orb}^{1}(G)$ induces a bijective correspondence between $\overline{\mathrm{YM}}\left(S^{2}, G\right)$ and $\operatorname{Orb}^{1}(G)$.

Proof. The assertion follows from the relation $\Omega_{f^{*} Z}=f^{*} \Omega_{Z}$ for any gauge transformation $f$ and from the correspondence between $\overline{\operatorname{Hom}}\left(S^{1}, G\right)$ and $\overline{\mathrm{YM}}\left(S^{2}, G\right)$, given in Sect. 2 .

Now we choose a maximal abelian subalgebra $t$ of $\mathfrak{g}$ and set $\mathcal{O}^{*}=\mathcal{O} \cap t$ for $\mathcal{O} \in \operatorname{Orb}(G)$. Using the fact that all maximal abelian subalgebras of the Lie algebra of a compact Lie group are conjugate, we obtain that $\mathcal{O}^{*}$ is an orbit of the action of the Weyl group on $t$. The distance $d\left(\mathcal{O}_{1}, \mathcal{O}_{2}\right)$ of two orbits $\mathcal{O}_{1}, \mathcal{O}_{2} \in \operatorname{Orb}(G)$ we define by means of the Ad-invariant scalar product on $g$, i.e.

$$
d\left(\mathcal{O}_{1}, \mathcal{O}_{2}\right)=\min \left\{\left\|X_{1}-X_{2}\right\| ; X_{1} \in \mathcal{O}_{1}, X_{2} \in \mathcal{O}_{2}\right\} .
$$

Similarly, we define

$$
d^{*}\left(\mathcal{O}_{1}^{*}, \mathcal{O}_{2}^{*}\right)=\min \left\{\left\|X_{1}-X_{2}\right\| ; X_{1} \in \mathcal{O}_{1}^{*}, X_{2} \in \mathcal{O}_{2}^{*}\right\} .
$$

Lemma 3.2. Let $\mathcal{O}_{1}, \mathcal{O}_{2} \in \operatorname{Orb}(G)$. Then $d\left(\mathcal{O}_{1}, \mathcal{O}_{2}\right)=d^{*}\left(\mathcal{O}_{1}^{*}, \mathcal{O}_{2}^{*}\right)$.

Proof. We choose $X_{1} \in \mathcal{O}_{1}$ and $X_{2} \in \mathcal{O}_{2}$ such that $X_{1} \in t$ and $d\left(\mathcal{O}_{1}, \mathcal{O}_{2}\right)=\left\|X_{1}-X_{2}\right\|$. It suffices to show $X_{2} \in t$. Since $\left\|X_{1}-X_{2}\right\|^{2} \leqq\left\|\operatorname{Ad}(g) X_{1}-X_{2}\right\|^{2}$ is valid for all $g \in G$, the unity of $G$ is a critical point of the mapping $f: g \in G \rightarrow\left\|\operatorname{Ad}(g) X_{1}-X_{2}\right\|^{2}$. 
Consequently,

$$
d f_{1}(Y)=-2\left\langle Y,\left[X_{1}, X_{2}\right]\right\rangle=0
$$

for all $Y \in g$. Hence $\left[X_{1}, X_{2}\right]=0$. Since $t$ is maximal abelian, $X_{2} \in t$ follows.

Let $\theta_{k}: t \rightarrow R, k=1, \ldots, l$ be the roots of $G$ and let $\gamma_{k}=S_{k}(0)$, where $S_{k}$ is the reflection of $t$ with respect to the hyperplane $\theta_{k}=1$. Denote by $\Gamma_{0}$ the additive group generated by the vectors $\gamma_{k}, k=1, \ldots, l$. It is clear that $\Gamma_{0}$ is a subgroup of the lattice $I=\{X \in t ; \exp X=1\}$.

Proposition 3.2. Let $\left(P_{1}, Z_{1}\right)$ and $\left(P_{2}, Z_{2}\right)$ be contained in $\mathrm{YM}\left(S^{2}, G\right)$. Then the bundles $P_{1}$ and $P_{2}$ are equivalent if and only if $\operatorname{vol}\left(S^{2}\right)\left(\mathcal{O}_{Z_{1}}^{*}-\mathcal{O}_{Z_{2}}^{*}\right) \subseteq \Gamma_{0}$.

Proof. Let $X_{1} \in \mathcal{O}_{Z_{1}}^{*}$ and $X_{2} \in \mathcal{O}_{Z_{2}}^{*}$ and consider the closed geodesics

$$
\varrho_{i}: t \in[0,1] \rightarrow \exp \left(t \cdot X_{i} \cdot \operatorname{vol}\left(S^{2}\right)\right) \in G, \quad i=1,2 .
$$

From [13], Theorem 18.3, we obtain that the bundles $P_{1}$ and $P_{2}$ are equivalent if and only if $\Phi\left(Z_{1}\right)$ and $\Phi\left(Z_{2}\right)$ are homotopic (see also the proof of Proposition 1.3). Applying the Propositions 2.1 and 3.1 and Corollary 2.1, we see that $\Phi\left(Z_{i}\right)$ and $\varrho_{i}$ $(i=1,2)$ are conjugate. Since $\varrho_{1}$ and $\varrho_{2}$ are homotopic if and only if $\operatorname{vol}\left(S^{2}\right)\left(X_{1}-X_{2}\right) \in \Gamma_{0}$ (see [1], 5.47), the lemma is proved.

Let $a=\min \left\{\|X\| ; X \in \Gamma_{0}, X \neq 0\right\}$. Because of $\Gamma_{0} \subseteq I$ we have $a>0$.

Now we formulate the isolation theorem.

Theorem 3.1. Let $Z_{1}$ and $Z_{2}$ be two Yang-Mills connections on a G-principal fibre bundle $P$ over $S^{2}$.

If $\operatorname{dist}\left(Z_{1}, Z_{2}\right)<a \cdot \operatorname{vol}\left(S^{2}\right)^{-1 / 2}$ holds, then the connections $Z_{1}$ and $Z_{2}$ are gauge equivalent.

Proof. Using Proposition 3.1, $\operatorname{dist}\left(Z_{1}, Z_{2}\right)<a \cdot \operatorname{vol}\left(S^{2}\right)$ implies

$$
\begin{aligned}
\operatorname{vol}\left(S^{2}\right)^{2} d\left(\mathcal{O}_{Z_{1}}, \mathcal{O}_{Z_{2}}\right)^{2} & =\operatorname{vol}\left(S^{2}\right) \cdot \int_{S^{2}} d\left(\mathcal{O}_{Z_{1}}, \mathcal{O}_{Z_{2}}\right)^{2} d S^{2} \\
& \leqq \operatorname{vol}\left(S^{2}\right) \cdot \inf _{f_{1}, f_{2} \in \mathscr{G}(P)} \int_{S^{2}}\left\|* \Omega_{f_{1}^{*} Z_{1}}-* \Omega_{f_{2}^{*} Z_{2}}\right\|^{2} d S^{2} \\
& =\operatorname{vol}\left(S^{2}\right) \cdot \inf _{f_{1}, f_{2} \in \mathscr{G}(P)} \int_{S^{2}}\left\|\Omega_{f_{1}^{*} Z_{1}}-\Omega_{f_{2}^{*} Z_{2}}\right\|^{2} d S^{2} \\
& =\operatorname{vol}\left(S^{2}\right) \cdot \operatorname{dist}\left(Z_{1}, Z_{2}\right)^{2}<a^{2} .
\end{aligned}
$$

Then, by Lemma 3.2, $\operatorname{vol}\left(S^{2}\right) d^{*}\left(\mathcal{O}_{Z_{1}}^{*}, \mathcal{O}_{Z_{2}}^{*}\right)<a$ holds. Furthermore, by Proposition 3.2, we have $\operatorname{vol}\left(S^{2}\right)\left(\mathcal{O}_{Z_{1}}^{*}-\mathcal{O}_{Z_{2}}^{*}\right) \subseteq \Gamma_{0}$. Since $a=\min \left\{\|X\| ; X \in \Gamma_{0}, X \neq 0\right\}$, we obtain $\mathcal{O}_{Z_{1}}=\mathcal{O}_{Z_{2}}$, and our statement follows from Proposition 3.1.

Corollary 3.1. Let $Z_{1}$ be a Yang-Mills connection on a G-principal fibre bundle P. Then

$$
\left\{Z \bmod \mathscr{G}(P) \in \mathscr{C}(P) / \mathscr{G}(P) ; \operatorname{dist}\left(Z_{1}, Z\right)<a \cdot \operatorname{vol}\left(S^{2}\right)^{-1 / 2}\right\}
$$

is a neighbourhood of $Z_{1} \bmod \mathscr{G}(P)$, in which no other equivalence classes of YangMills connections appear.

Proof. This follows from Theorem 3.1 and the relation $\operatorname{dist}\left(f^{*} Z, \bar{f}^{*} \bar{Z}\right)$ $=\operatorname{dist}(Z, \bar{Z})$ for $Z, \bar{Z} \in \mathscr{C}(P)$ and $f, \bar{f} \in \mathscr{G}(P)$. 


\section{References}

1. Adams, J.: Lectures on Lie groups. New York, Amsterdam: Benjamin 1969

2. Atiyah, M.F., Bott, R.: The Yang-Mills equation over Riemann surfaces. Phil. Trans. Roy. Soc. (London) A 308, 523 (1982)

3. Bourguignon, J.P., Lawson, H.B., Jr.: Stability and isolation phenomena for Yang-Mills fields. Commun. Math. Phys. 79, 189-230 (1981)

4. Coleman, S.: The magnetic monopole fifty years later. Proc. of the 19th Intern. School of Subnuclear Physics, p. 21. Zichichi, A., Erice 1981

5. Forster, O.: Lectures on Riemann surfaces. Berlin, Heidelberg, New York: Springer 1982

6. Goddard, P., Nuyts, J., Olive, D.: Gauge theories and magnetic charge. Nucl. Phys. B 125, $1-28$ (1977)

7. Klingenberg, W.: Riemannian geometry. Berlin, New York: de Gruyter 1982

8. Kobayashi, S., Nomizu, K.: Foundations of differential geometry, Vol. 1. New York, London: Interscience 1963

9. Kostant, B.: Quantization and unitary representations. Lecture Notes in Mathematics, Vol. 170, pp. 87-207. Berlin, Heidelberg, New York: Springer 1980

10. Minami, M.: Dirac's Monopole and the Hopf Map. Prog. Theor. Phys. 62, 1128 (1979)

11. Mitter, P.K., Viallet, C.M.: On the bundle of connections and the gauge orbit manifold in Yang-Mills theory. Commun. Math. Phys. 79, 457-472 (1981)

12. Singer, I.M.: The geometry of the orbit space for non-abelian gauge theories. Phys. Scr. 24, $817-820(1981)$

13. Steenrod, N.: The topology of fibre bundles. Princeton, NS: Princeton University Press 1951

14. Sulanke, R., Wintgen, P.: Differentialgeometrie und Faserbündel. Berlin: Deutscher Verlag der Wissenschaften 1972

Communicated by S. W. Hawking

Received August 8, 1984; in revised form April 5, 1985 
